\title{
HILBERT-ASAI EISENSTEIN SERIES, REGULARIZED PRODUCTS, AND HEAT KERNELS
}

\author{
JAY JORGENSON AND SERGE LANG
}

\begin{abstract}
In a famous paper, Asai indicated how to develop a theory of Eisenstein series for arbitrary number fields, using hyperbolic 3-space to take care of the complex places. Unfortunately he limited himself to class number 1 . The present paper gives a detailed exposition of the general case, to be used for many applications. First, it is shown that the Eisenstein series satisfy the authors' definition of regularized products satisfying the generalized Lerch formula, and the basic axioms which allow the systematic development of the authors' theory, including the Cramér theorem. It is indicated how previous results of Efrat and Zograf for the strict Hilbert modular case extend to arbitrary number fields, for instance a spectral decomposition of the heat kernel periodized with respect to $\mathrm{SL}_{2}$ of the integers of the number field. This gives rise to a theta inversion formula, to which the authors' Gauss transform can be applied. In addition, the Eisenstein series can be twisted with the heat kernel, thus encoding an infinite amount of spectral information in one item coming from heat Eisenstein series. The main expected spectral formula is stated, but a complete exposition would require a substantial amount of space, and is currently under consideration.
\end{abstract}

The Hilbert modular case for totally real number fields has been well understood for many decades. Asai gave a beautiful treatment showing how to deal with the general case [As 70], but unfortunately he limited himself to number fields of class number one. As far as a general exposition is concerned, matters were not much improved in [EGM 85], which limited itself to imaginary quadratic fields. Of course, [EGM 87] then pushed matters in a deeper way in the direction of special values of Eisenstein series.

As shown below, a general exposition of the Eisenstein series for arbitrary number fields turns out not to be more difficult without any restriction and doing so actually forces a clarification of the terminology and the notation. We shall deal with applications in a different direction from that

Received May, 19, 1996.

Jorgenson acknowledges support from NSF Grants, from the Institute for Advanced Study, and from a Sloan Fellowship. Lang thanks the Max-Planck-Institut for productive yearly visits. 
of [EGM 85]. Asai ran across theta-type series formed with the $K$-Bessel function, aside from the Eisenstein series. Bessel functions play a dual role. On the one hand, they give rise to series playing the role of Dirichlet series. In [JoL 96], we showed that taking the Gauss transform of ordinary theta series yields Bessel series in lieu of Dirichlet series, and we showed how these Bessel series fit in our general framework of regularized products. Here we shall first take the usual Mellin transform of Bessel theta series to get the corresponding Dirichlet series. As mentioned in [JoL 96], we leave to another paper the fuller theory which arises from taking the Gauss transform of the Bessel series in general, yielding Legendre series. The tabulations of the present paper will be useful in this subsequent work.

In $\S 1$ and $\S 2$, we carry out the Riemann-Hecke arguments for a functional equation in a fairly broad context, applying to Bessel theta series. Independently, in $\S 3, \S 4$ and $\S 5$ we formulate the general properties of Hilbert-Asai Eisenstein series. In $\S 6$, we show how the Eisenstein series fit in the theory of regularized products as developed beginning in the articles [JoL 93] and [JoL 94]. In $\S 7$, we show how the heat kernel on the symmetric space associated to the number field can be defined in terms of the Eisenstein series, so that we can apply the general theory and especially [JoL 96]. Finally, in $\S 8$, we reformulate the results of $\S 7$ in terms of a new Eisenstein series which we call the heat Eisenstein series, thus naturally leading to work in progress concerning spectral expansion on other symmetric spaces.

Thus we can take the Gauss transform of the heat kernel inversion formula to obtain new zeta functions with functional equations. In the case of Riemann surfaces, these functions correspond to logarithmic derivatives of Selberg zeta functions.

Among other things, the present paper provides significant examples for the general theory of regularized products and series. We note that these examples also serve to emphasize the sufficiency of a Dirichlet series representation, as opposed to the more classical emphasis on the existence of Euler products as such. So far, Euler products have not played a role in the general theory we are developing. 


\section{$\S 1$. Functional equation and Mellin transform on a product of $G_{m}^{+}$}

Let $S=\{v\}$ be a finite set and let

$$
Y=\prod_{v \in S} Y_{v} \quad \text { where } \quad Y_{v}=\mathbf{R}^{+} .
$$

Let

$$
d^{*} y_{v}=\frac{d y_{v}}{y_{v}} \quad \text { and } \quad d^{*} y=\prod_{v \in S} \frac{d y_{v}}{y_{v}} .
$$

For each $v$ we let $N_{v}$ be a positive real number and we let $N=\sum N_{v}$. We define the norm

$$
\mathbf{N} y=\prod_{v \in S} y_{v}^{N_{v}}
$$

Thus the norm $\mathbf{N}: Y \rightarrow \mathbf{R}^{+}$is a continuous homomorphism. We let

$$
Y^{0}=\operatorname{Ker} \mathbf{N}=\left\{y \in Y \text { such that } \prod_{v \in S} y_{v}^{N_{v}}=1\right\} .
$$

Let $U$ be an abelian group with a fixed homomorphism onto a discrete subgroup $V$ of $Y^{0}$ such that $Y^{0} / V$ is compact. Since there is an isomorphism

$$
\log : Y \rightarrow \mathbf{R}^{\#(S)}
$$

our assumption on $U$ amounts to saying that $\log Y^{0}$ is a hyperplane in $\log Y$ and the image of $U$ in $\log Y^{0}$ is a lattice. Thus, $Y^{0} / V$ is isomorphic to a real torus of dimension $\#(S)-1$.

We note that $\mathbf{R}^{+}$acts on $Y$ and can even be embedded as a subgroup of $Y$, namely for $a \in \mathbf{R}^{+}$we let

$$
a y=\left(\ldots, a^{1 / N} y_{v}, \ldots\right)
$$

so $a$ is embedded as $\left(\ldots, a^{1 / N}, \ldots\right)$ in $Y$. Then $\mathbf{N} a=a$. This embedding splits the sequence

$$
0 \rightarrow Y^{0} \rightarrow Y \rightarrow \mathbf{R}^{+} \rightarrow 0
$$

so an element $y \in Y$ can be written uniquely in the form $y=t y^{0}$ with $t \in \mathbf{R}^{+}, y^{0} \in Y^{0}$.

The measure $d^{*} y$ determines a measure on $Y / V$. It can also be written as a product measure

$$
d^{*} y=d^{*} y^{0} \frac{d t}{t}
$$


where $y^{0}$ is the variable in $Y^{0}$, and $d^{*} y^{0}$ is a Haar measure on $Y^{0}$, uniquely determined by $d^{*} y$.

Before carrying out a general theorem, we recall two distinct special cases arising in classical situations. Let us call a pair of functions $h, h_{0}$ on $Y$ admissible if they satisfy the conditions:

ADM 1: $h, h_{0}$ are invariant under the action of $V$, so are defined on $Y / V$;

ADM 2: $h_{0}$ extends continuously to $Y^{0} / V \times[0, \infty)$;

ADM 3: $h_{0}(y)$ decreases exponentially as $\mathbf{N} y \rightarrow \infty$.

For algebraic number fields: The function $h$ satisfies the inversion conditions:

INV 1: (Number Fields) $h(y)=h_{0}(y)+c_{0}$ for some constant $c_{0}$;

INV 2: (Number Fields) $\mathbf{N} y^{-\frac{1}{2}} h\left(y^{-1}\right)=h(y)$.

For Eisenstein series as in [As 70], see also Theorem 4.4 below.

INV 1: (Eisenstein) $h(y)=h_{0}(y)+c_{0} \mathbf{N} y$;

INV 2: (Eisenstein) $h\left(y^{-1}\right)+\log \mathbf{N} y=h(y)$.

We define the Mellin transform on $Y / V$, with respect to $\mathbf{N}$ :

$$
\mathbf{M}_{Y / V} h_{0}(s)=\int_{Y / V} h_{0}(y) \mathbf{N} y^{s} d^{*} y .
$$

The next theorem covers the special case considered in Asai [As 70].

THEOREM 1.1. Under the Eisenstein conditions INV 1, INV 2 above, the function $\mathbf{M}_{Y / V} h_{0}$ has a meromorphic continuation and satisfies the functional equation

$$
\mathbf{M}_{Y / V} h_{0}(-s)=\mathbf{M}_{Y / V} h_{0}(s) .
$$

The above theorem extends easily to the case when we include a character as follows. By a Hecke character $\chi$ on $Y$, with respect to the given action of $V$ and $\mathbf{R}^{+}$, we mean a continuous homomorphism

$$
\chi: Y \longrightarrow \mathbf{C}^{1}
$$

into the unit circle, such that $\chi$ is $\mathbf{R}^{+}$- and $V$-invariant, that is,

$$
\chi(a y)=\chi(u y)=\chi(y)
$$


for all $y \in Y, a \in \mathbf{R}^{+}$and $u \in V$. Then $\chi$ induces a character on $Y^{0} / V$, and since $Y^{0} / V$ is a real torus, it follows that the group of Hecke characters is isomorphic to $\mathbf{Z}^{r}$, where $r=\#(S)-1$.

For a Hecke character $\chi$, we define

$$
\mathbf{M}_{Y / V} h_{0}(s, \chi)=\int_{Y / V} h_{0}(y) \chi(y) \mathbf{N} y^{s} d^{*} y
$$

Then one obtains the functional equation with Hecke characters:

THEOREM 1.2. Under the Eisenstein conditions, assume that $\chi$ is nontrivial. Then

$$
\mathbf{M}_{Y / V} h_{0}(s, \chi)=\int_{\mathbf{N} y \geqq 1} h_{0}(y)\left[\chi(y) \mathbf{N} y^{s}+\bar{\chi}(y) \mathbf{N} y^{-s}\right] d^{*} y
$$

In particular,

$$
\mathbf{M}_{Y / V} h_{0}(s, \chi)=\mathbf{M}_{Y / V} h_{0}(-s, \bar{\chi})
$$

Recall that a quasi character

$$
Y / V \longrightarrow \mathbf{C}^{*}
$$

is simply a continuous homomorphism. It is therefore uniquely determined by a vector $\beta=\left(\ldots, \beta_{v}, \ldots\right)$ of complex numbers, not necessarily of absolute value 1 , satisfying the orthogonality relations

OR 1. $\sum_{v \in S} \beta_{v} N_{v} \log u_{v} \equiv 0 \bmod 2 \pi i \mathbf{Z} \quad$ for all $u \in V$.

The value of the quasi character $[\beta]$ corresponding to $\beta$ is given by

$$
[\beta](y)=\prod_{v \in S} y_{v}^{N_{v} \beta_{v}}
$$

If we let $\chi$ be the Hecke character induced on $Y^{0} / V$, and such that $\chi\left(\mathbf{R}^{+}\right)=$ 1 , then there exists a unique complex number $s$ such that

$$
[\beta]\left(t y^{0}\right)=\chi\left(y^{0}\right) t^{s}, \text { that is }[\beta]=\chi \cdot \mathbf{N}^{s} .
$$

The quasi character is equal to the Hecke character if and only if we also have the orthogonality relation 
OR 2. $\sum_{v \in S} \beta_{v} N_{v}=0$.

Thus we say $\chi=[\beta]$ if and only if $\mathbf{O R} \mathbf{1}$ and $\mathbf{O R} \mathbf{2}$ are satisfied.

Under these two conditions, it follows that $\operatorname{Re}\left(\beta_{v}\right)=0$ for all $v$, that is $\beta_{v}$ is pure imaginary for all $v$, so evidently $[\beta]$ has values on the unit circle.

The above situation can be further generalized in a manner necessary for certain applications. For one thing, one need not deal only with the pair $\left(h, h_{0}\right)$, but two pairs can intervene. Furthermore, the powers of $\mathbf{N} y$ or $\log \mathbf{N} y$ need not be 1 . So we consider the following more general formulations of INV 1, INV 2. For this, we need the notion of generalized polynomials as they have appeared systematically in the [JoL] series, namely a finite sum

$$
P(T)=\sum c_{p, m}(\log T)^{m} T^{p}=\sum B_{p}(\log T) T^{p},
$$

with $p \in \mathbf{C}, c_{p, m} \in \mathbf{C}, m \in \mathbf{Z}_{\geqq 0}$, and a polynomial $B_{p}$ for each $p$. The conditions then read:

INV 1. There is a generalized polynomial $P(T)$ such that

$$
h(y)=h_{0}(y)+P(\mathbf{N} y) .
$$

and there are admissible functions $\tilde{h}, \tilde{h}_{0}$ such that

$$
\tilde{h}(y)=\tilde{h}_{0}(y)+\tilde{P}(\mathbf{N} y)
$$

for some generalized polynomial $\tilde{P}$.

INV 2. There is a generalized polynomial $Q$ and $s_{0} \in \mathbf{C}$ such that

$$
\mathbf{N} y^{-s_{0} / 2} \tilde{h}\left(y^{-1}\right)+Q(\mathbf{N} y)=h(y) .
$$

Define the truncated Mellin transform of a generalized polynomial $P(T)$ as written above on the interval $[0,1]$ to be

$$
\mathbf{M}_{0}^{1} P(s)=\sum c_{p, m}\left(\frac{d}{d s}\right)^{m} \frac{1}{s+p}=\sum c_{p, m} \frac{(-1)^{m} m !}{(s+p)^{m+1}} .
$$


Lemma 1.3. Let $\operatorname{Re}(s+p)>0$ for all $p$ such that $c_{p, m} \neq 0$. Then

$$
\int_{\mathbf{N} y \leqq 1} P(\mathbf{N} y) \chi(y) \mathbf{N} y^{s} d^{*} y=\delta_{\chi} \mu^{*}\left(Y^{0} / V\right) \mathbf{M}_{0}^{1} P(s) .
$$

where $\delta_{\chi}=1$ if $\chi$ is trivial and 0 otherwise

Proof. Immediate.

We define

$$
P^{-}(T)=P\left(T^{-1}\right) .
$$

THEOREM 1.4. We have the meromorphic continuation

$$
\begin{aligned}
\mathbf{M}_{Y / V} h_{0}(s, \chi)= & \int_{\mathbf{N} y \geqq 1}\left[h_{0}(y) \chi(y) \mathbf{N} y^{s}+\tilde{h}_{0}(y) \bar{\chi}(y) \mathbf{N} y^{-s+s_{0} / 2}\right] d^{*} y \\
& +\delta_{\chi} \mu^{*}\left(Y^{0} / V\right) \mathbf{M}_{0}^{1}\left(Q+\tilde{P}^{-}-P\right)(s) .
\end{aligned}
$$

The integral is entire in $s$ and in particular

$$
\mathbf{M}_{Y / V} h_{0}(s, \chi)=M_{Y / V} \tilde{h}_{0}(-s, \bar{\chi})+\operatorname{Rat}(s),
$$

where $\operatorname{Rat}(s)$ is a rational function in $s$.

Proof. The proof follows a classical pattern and runs as follows:

$$
\begin{aligned}
\mathbf{M}_{Y / V} & h_{0}(s, \chi) \\
= & \int_{\mathbf{N} y \leqq 1}+\int_{\mathbf{N} y \geqq 1} h_{0}(y) \chi(y) \mathbf{N} y^{s} d^{*} y \\
= & \int_{\mathbf{N} y \leqq 1} h(y) \chi(y) \mathbf{N} y^{s} d^{*} y-\int_{\mathbf{N} y \leqq 1} P(\mathbf{N} y) \chi(y) \mathbf{N} y^{s} d^{*} y \\
& \quad+\int_{\mathbf{N} y \geqq 1} h_{0}(y) \chi(y) \mathbf{N} y^{s} d^{*} y \\
= & \int_{\mathbf{N} y \leqq 1} \mathbf{N} y^{-s_{0} / 2} \tilde{h}\left(y^{-1}\right) \chi(y) \mathbf{N} y^{s} d^{*} y+\int_{\mathbf{N} y \leqq 1} Q(y) \chi(y) \mathbf{N} y^{s} d^{*} y \\
& -\int_{\mathbf{N} y \leqq 1} P(\mathbf{N} y) \chi(y) \mathbf{N} y^{s} d^{*} y+\int_{\mathbf{N} y \geqq 1} h_{0}(y) \chi(y) \mathbf{N} y^{s} d^{*} y .
\end{aligned}
$$

First integral on right 


$$
\begin{aligned}
& =\int_{\mathbf{N} y \geqq 1} \tilde{h}(y) \overline{\chi(y)} \mathbf{N} y^{-s+s_{0} / 2} d^{*} y \\
& =\int_{\mathbf{N} y \geqq 1} \tilde{h}_{0}(y) \overline{\chi(y)} \mathbf{N} y^{-s+s_{0} / 2} d^{*} y+\int_{\mathbf{N} y \geqq 1} \tilde{P}(\mathbf{N} y) \overline{\chi(y)} \mathbf{N} y^{-s} d^{*} y \\
& =\int_{\mathbf{N} y \geqq 1} \tilde{h}_{0}(y) \overline{\chi(y)} \mathbf{N} y^{-s+s_{0} / 2} d^{*} y+\int_{\mathbf{N} y \leqq 1} \tilde{P}^{-}(\mathbf{N} y) \chi(y) \mathbf{N} y^{s} d^{*} y .
\end{aligned}
$$

Putting (1) and (2) together yields the asserted result.

Note that Theorem 1.4 covers both Theorem 1.1 and Theorem 1.2, except that the formulations in the previous theorems are simpler because the rational function disappears due to the simpler conditions on $h$ and $h_{0}$.

\section{$\S 2$. Bessel theta series and Mellin transforms}

In this section we see how one can form Bessel series which play the role of theta series.

We let $K_{\alpha}$ be the $K$-Bessel function, with $\alpha \in \mathbf{C}$, which is normalized as in [La 73/87] and [JoL 96], that is, for $c>0$ :

$$
K_{\alpha}(c)=\int_{0}^{\infty} e^{-c(t+1 / t)} t^{\alpha} \frac{d t}{t} .
$$

If $K_{\alpha}^{B}$ denotes the one found in classical tables, then

$$
2 K_{\alpha}^{B}(2 c)=K_{\alpha}(c)
$$

We suppose given a real number $\sigma_{1}>0$. We suppose that $h_{0}$ is a function which can be expressed as a Bessel theta series, meaning the following. There are constants $a_{k} \in \mathbf{C}, c_{k, v} \in \mathbf{R}^{+}, \alpha_{v} \in \mathbf{C}$ such that

$$
h_{0}(y)=\sum_{k=1}^{\infty} a_{k} \sum_{v \in V} \prod_{v} K_{\alpha_{v}}\left(c_{k, v} y_{v}\right) y_{v}^{N_{v} \sigma_{1}} .
$$

satisfies the Bessel-theta convergence condition:

B-TH. There is a number $\sigma_{0}^{\prime} \geqq 0$ such that for $\operatorname{Re}(s)>\sigma_{0}^{\prime}$ the series

$$
\sum_{k=1}^{\infty}\left|a_{k}\right| \mathbf{N} c_{k}^{-\left(s+\sigma_{1}\right)}
$$


converges absolutely and thus uniformly in any half plane $\operatorname{Re}(s) \geqq$ $\sigma_{0}^{\prime}+\varepsilon$.

Of course, we put

$$
c_{k}=\left(\ldots, c_{k, v}, \ldots\right) \quad \text { and } \quad \mathbf{N} c_{k}=\Pi_{v} c_{k, v}^{N_{v}} .
$$

Note that the condition $\sigma_{1}>0$ implies that $h_{0}$ extends continuously to the space $Y^{0} \times[0, \infty)$, and $h_{0}$ is invariant under the action of $V$. Furthermore, from K7 of [La 73/87], Chapter 20, $\S 3$, it follows that $h_{0}(y)$ is exponentially decreasing for $\mathbf{N} y \rightarrow \infty$, so $h_{0}$ is admissible.

By a standard Bessel indentity, we know that for $\operatorname{Re}(s)>|\operatorname{Re}(\alpha)|$,

$$
\int_{0}^{\infty} K_{\alpha}(c t) t^{s} \frac{d t}{t}=\frac{1}{2} c^{-s} \Gamma\left(\frac{s-\alpha}{2}\right) \Gamma\left(\frac{s+\alpha}{2}\right) .
$$

A proof follows by the same technique as in [La 73], Chapter $20, \S 3$.

Since

$$
\int_{Y}=\int_{Y / V} \sum_{V} \quad \text { and } \quad d^{*} y=d^{*} y^{0} \frac{d t}{t}
$$

the above Bessel identity immediately allows us to compute the Mellin transform of the Bessel series on $Y / V$. We assume that $d^{*} y$ is normalized the usual way,

$$
d^{*} y=\prod \frac{d y_{v}}{y_{v}} .
$$

Then for a Hecke character $\chi=[\beta]$, remembering that OR 1 and OR 2 are satisfied, we get

$$
\mathbf{M}_{Y / V} h_{0}((s, \chi))=\sum_{k=1}^{\infty} a_{k} \prod_{v} \int_{Y_{v}} K_{\alpha_{v}}\left(c_{k, v} y_{v}\right) y_{v}^{N_{v} \sigma_{1}} y_{v}^{N_{v} s} y_{v}^{N_{v} \beta_{v}} \frac{d y_{v}}{y_{v}} .
$$

We have

$$
\chi\left(c_{k}\right)=\prod_{v} c_{k, v}^{n_{v} \beta_{v}} .
$$

We let

$$
s_{v}=N_{v}\left(s+\sigma_{1}+\beta_{v}\right) .
$$

We then obtain: 
Theorem 2.1. Let $Z(s)$ be the Dirichlet series

$$
Z(s)=\sum_{k=1}^{\infty} \frac{2^{-\#(S)} \chi\left(c_{k}^{-1}\right) a_{k}}{\mathbf{N} c_{k}^{s+\sigma_{1}}}
$$

and let

$$
\begin{aligned}
G_{v}(s) & =\Gamma\left(\frac{1}{2}\left(s_{v}-\alpha_{v}\right)\right) \Gamma\left(\frac{1}{2}\left(s_{v}+\alpha_{v}\right)\right) \\
G(s) & =\prod_{v} G_{v}\left(s_{v}\right) .
\end{aligned}
$$

Then for $\operatorname{Re}(s)>\sigma_{0}^{\prime}$,

$$
\mathbf{M}_{Y / V} h_{0}(s, \chi)=G(s) Z(s) .
$$

Remark. When $\alpha=1 / 2$ then $K_{1 / 2}$ collapses to the exponential function and the Gauss duplication formula shows that the product of the two gamma factors actually collapses to one gamma factor. This is precisely what happens in the most classical case of the Dedekind zeta function of number fields.

Theorems 1.1 and 1.2 apply to the Bessel series which will be defined below, but the rest of this paper is logically independent of what precedes.

For the convenience of the reader, we now recall Hecke's functional equation for the Dedekind zeta function of a number field $F$, to be used below. We let $\mathbf{D}_{F}$ denote the absolute value of the discriminant and we let $\mathfrak{K}$ denote an ideal class. We let $\mathfrak{d}$ be the different. As usual, $r_{1}$ and $r_{2}$ denote the number of real resp. complex conjugate embeddings of $F$. We recall the classical zeta function associated with a fractional ideal $\mathfrak{a} \neq(0)$, namely

$$
\zeta(s, \mathfrak{a})=\mathbf{N a}^{s} \sum_{(\mu)} \mathbf{N}(\mu)^{-s},
$$

where $\mathbf{N}$ is the absolute norm and the sum is taken over all principal ideals $(\mu) \subset \mathfrak{a}$ with $\mu \neq 0$. Because of the factor $\mathbf{N a}^{s}$, one sees that $\zeta(s, \mathfrak{a})$ depends only on the ideal class of $\mathfrak{a}$. If $\mathfrak{a} \in \mathfrak{K}^{-1}$ with an ideal class $\mathfrak{K}$, we set

$$
\zeta(s, \mathfrak{a})=\zeta(s, \mathfrak{K}) .
$$


TheOREM 2.2. (Hecke functional equation) Let $G$ be the function

$$
\begin{aligned}
G(s)=G_{F}(s) & =\mathbf{D}_{F}^{s / 2}\left(\pi^{-s / 2} \Gamma(s / 2)\right)^{r_{1}}\left((2 \pi)^{-s} \Gamma(s)\right)^{r_{2}} \\
& =A^{s / 2} \Gamma(s / 2)^{r_{1}} \Gamma(s)^{r_{2}}
\end{aligned}
$$

where $A=\mathbf{D}_{F} \pi^{-r_{1}}(2 \pi)^{-2 r_{2}}$. Let

$$
\xi(s, \mathfrak{K})=G(s) \zeta(s, \mathfrak{K}) .
$$

Let $\mathfrak{K}^{\prime}$ be the dual class, i.e. the ideal class $\mathfrak{d}^{-1} \mathfrak{K}^{-1}$. Then $\xi(s, \mathfrak{K})$ is holomorphic in $s$ except for simple poles at $s=0$ and $s=1$ and

$$
\xi(s, \mathfrak{K})=\xi\left(1-s, \mathfrak{K}^{\prime}\right) .
$$

\section{§3. Hyperbolic spaces, number fields and Eisenstein series}

Let $F$ be a number field of degree $N$ over $\mathbf{Q}$. We let $S=S_{\infty}$ be the set of absolute values of absolute values at infinity and to each $v \in S_{\infty}$ we suppose chosen a fixed embedding

$$
F \hookrightarrow \mathbf{R} \quad \text { or } \quad F \hookrightarrow \mathbf{C}
$$

according as $v$ is real or complex. We let $\mathfrak{o}=\mathfrak{o}_{v}$ be the ring of algebraic integers in $F$ at a finite place $v$ and we let $\mathfrak{d}=\mathfrak{d}_{\mathfrak{o} / \mathbf{Z}}$ be the different as above.

We need certain spaces from differential geometry. We let:

$$
\begin{aligned}
& \mathbf{h}_{2}=\text { upper half plane }=\mathbf{R} \times \mathbf{R}^{+} \text {with its usual Poincaré metric. } \\
& \mathbf{h}_{3}=\text { hyperbolic } 3 \text {-space }=\mathbf{C} \times \mathbf{R}^{+}, \text {on which we make more comments. }
\end{aligned}
$$

The unique simply connected Riemannian manifold of dimension 3, with constant negative curvature -1 , up to isometry, has many models, of which the following is the relevant one, as described in Kubota [Ku 68]. We let $\mathbf{h}_{3}$ be the space of matrices

$$
z=\left(\begin{array}{cc}
x & -y \\
y & \bar{x}
\end{array}\right) \quad \text { with } x \in \mathbf{C} \text { and } y \in \mathbf{R}, y>0 .
$$

We then write $y=y(z)$.

The group $\mathrm{SL}_{2}(\mathbf{C})$ operates on $\mathbf{h}_{3}$ in a natural way as follows. Let

$$
\sigma=\left(\begin{array}{cc}
\alpha & \beta \\
\gamma & \delta
\end{array}\right) \in \mathrm{SL}_{2}(\mathbf{C})
$$


Let $\alpha$ be a complex number. We write

$$
\tilde{\alpha}=\left(\begin{array}{cc}
\alpha & 0 \\
o & \bar{\alpha}
\end{array}\right)
$$

We define

$$
\sigma\langle z\rangle=(\tilde{\alpha} z+\tilde{\beta})(\tilde{\gamma} z+\tilde{\delta})^{-1} .
$$

Quite generally, let $\gamma, \delta \in \mathbf{C}$ not both 0 . For $z \in \mathbf{h}_{3}$ define

$$
y(\gamma, \delta ; z)=\frac{y(z)}{|\gamma x+\delta|^{2}+|\gamma|^{2} y^{2}}
$$

Then a straightforward calculation shows that

$$
y(\sigma\langle z\rangle)=y(\gamma, \delta ; z) .
$$

This is the analogue of the standard formula for the imaginary part of the image of a complex number under an element of $\mathrm{SL}_{2}(\mathbf{R})$.

Having the above simple notions we relate them to the number field $F$ as follows. We let:

$$
\begin{aligned}
\mathbf{h}_{v} & =\mathbf{h}_{2} \text { if } v \text { is real, and }=\mathbf{h}_{3} \text { if } v \text { is complex. } \\
\mathbf{h}_{F} & =\prod_{v \in S_{\infty}} \mathbf{h}_{v} .
\end{aligned}
$$

An element $z$ of $\mathbf{h}_{F}$ is thus a vector, $z=\left(\ldots, z_{v}, \ldots\right)$, and

$$
z_{v}=\left(x_{v}, y_{v}\right)
$$

For $v$ real, $x_{v} \in \mathbf{R}$ and for $v$ complex, $x_{v} \in \mathbf{C}$. In both cases, $y_{v} \in \mathbf{R}^{+}$.

We define $y(z)$ to be the vector

$$
y(z)=\left(\ldots, y\left(z_{v}\right), \ldots\right)_{v \in S_{\infty}} .
$$

We define the Norm

$$
\mathbf{N} y(z)=\prod_{v \in S_{\infty}} y\left(z_{v}\right)^{\mathbf{N}_{v}},
$$

where $\mathbf{N}_{v}=1$ or 2 according as $v$ is real or complex.

If $\mu, \nu \in F$ are not both 0 and $z \in \mathbf{h}_{F}$, then we put

$$
y(\mu, \nu ; z)=\left(\ldots, \frac{y_{v}}{\left|\mu_{v} x_{v}+\nu_{v}\right|^{2}+\left|\mu_{v}\right|^{2} y_{v}^{2}}, \ldots\right)
$$


where $y_{v}=y\left(z_{v}\right)$.

Given a pair of elements $(\mu, \nu)$ in $F \times F$ not both 0 we define the equivalence class

$$
\{\mu, \nu\}
$$

to consist of all pairs $\left(\mu_{1}, \nu_{1}\right)$ such that there exists a unit $\epsilon$ for which

$$
\left(\mu_{1}, \nu_{1}\right)=\epsilon(\mu, \nu)=(\epsilon \mu, \epsilon \nu)
$$

Let $\mathfrak{a}$ be a fractional ideal $\neq 0$. We define:

$$
\begin{aligned}
\operatorname{Equ}(\mathfrak{a}) & =\text { equivalence classes of pairs }\{\mu, \nu\} \text { with } \mu, \nu \in \mathfrak{a} \\
\operatorname{Equ}^{*}(\mathfrak{a}) & =\text { equivalence classes of pairs }\{\mu, \nu\} \text { with }(\mu, \nu)=\mathfrak{a} .
\end{aligned}
$$

We define the Eisenstein series

$$
E(z, s, \mathfrak{a})=\sum_{\{\mu, \nu\} \in \operatorname{Equ}(\mathfrak{a})} \mathbf{N} y(\mu, \nu ; z)^{s} \mathbf{N a}^{2 s}
$$

Thus the Eisenstein series is a higher dimensional version of the zeta series $\zeta(s, \mathfrak{a})$. Like the zeta series, the Eisenstein series converges absolutely for $\operatorname{Re}(s)>1$.

Let $\mathfrak{K}$ be an ideal class of $F$. Let $\mathfrak{a} \in \mathfrak{K}^{-1}$ be a fractional ideal. We define the $\mathfrak{K}$-Eisenstein series

$$
E(z, s, \mathfrak{K})=E(z, s, \mathfrak{a})
$$

The fact that we put a factor $\mathbf{N a}^{2 s}$ in the definition of the Eisenstein series shows immediately that the series depend only on the ideal class of $\mathfrak{a}$, or that of $\mathfrak{a}^{-1}$. We now define the primitive Eisenstein series

$$
E^{*}(z, s, \mathfrak{K})=\sum_{\{\mu, \nu\}=\mathfrak{a}} \mathbf{N} y(\mu, \nu ; z)^{s} \mathbf{N} \mathfrak{a}^{2 s}
$$

where the sum is taken over all equivalence classes of pairs

$$
\{\mu, \nu\} \in \mathrm{Equ}^{*}(\mathfrak{a})
$$

namely such that $(\mu, \nu)$ generates precisely the ideal a. Thus $E^{*}(z, s, \mathfrak{K})$ is a partial sum of the complete Eisenstein series $E(z, s, \mathfrak{K})$. Immediately 
from the definition, since a fractional ideal $(\mu, \nu)$ in $\mathfrak{a}$ can be written in the form $\mathfrak{a} \mathfrak{b}$ with some ideal $\mathfrak{b} \subset \mathfrak{o}$, we obtain

$$
E(z, s, \mathfrak{K})=\sum_{\mathfrak{b}} \frac{\mathfrak{E}^{*}(\mathfrak{z}, \mathfrak{s}, \mathfrak{a} \mathfrak{b})}{\mathbf{N b}^{2 \mathfrak{s}}}
$$

where the sum is taken over all ideals $\mathfrak{b} \neq 0$ in $\mathfrak{o}$. Since $E^{*}(z, s, \mathfrak{a} \mathfrak{b})$ depends only on the ideal class of $\mathfrak{a} \mathfrak{b}$, which is $\mathfrak{b} \mathfrak{K}^{-1}$, we therefore obtain the relation

$$
\begin{aligned}
E(z, s, \mathfrak{K}) & =\sum_{\mathfrak{L}} \sum_{\mathfrak{b} \in \mathfrak{L}} \mathbf{N b}^{-2 s} E^{*}\left(z, s, \mathfrak{L}^{-1} \mathfrak{K}\right) \\
& =\sum_{\mathfrak{L}} \zeta(2 s, \mathfrak{L}) E^{*}\left(z, s, \mathfrak{L}^{-1} \mathfrak{K}\right) .
\end{aligned}
$$

Summing over $\mathfrak{K}$, let us define the total Eisenstein series, independent of the class, by

$$
E_{F}(z, s)=\sum_{\mathfrak{K}} E(z, s ; \mathfrak{K}) \quad \text { and } \quad E_{F}^{*}(z, s)=\sum_{\mathfrak{K}} E^{*}(z, s ; \mathfrak{K}) .
$$

Since $\sum_{\mathfrak{L}} \zeta(s, \mathfrak{L})=\zeta_{F}(s)$, we obtain

$$
E_{F}(z, s)=\zeta_{F}(2 s) E_{F}^{*}(z, s)
$$

Thus the zeta function of $F$ appears as a natural factor of the Eisenstein series.

We shall derive a meromorphic continuation for $E(z, s, \mathfrak{a})$, by a certain inversion of theta series. We shall thus be led to a dual notion of equivalence of pairs, as follows. For $\mu, \nu^{\prime} \in F$ and $\mu \nu^{\prime} \neq 0$, we define $\left[\mu, \nu^{\prime}\right]=$ equivalence class of pairs under the equivalence relation

$$
\left(\mu, \nu^{\prime}\right) \sim\left(\epsilon \mu, \epsilon^{-1} \nu^{\prime}\right)
$$

for all units $\epsilon$. Observe that the product $\mu \nu^{\prime}$ depends only on the class.

We shall obtain a Bessel series for $E(z, s, \mathfrak{a})$. For this purpose, with $\mu, \nu^{\prime} \in F$ and $\mu \nu^{\prime} \neq 0$ we let the $v$-Bessel factor be

$$
\begin{aligned}
B_{v}\left(\mu, \nu^{\prime}, y, s\right) & =y_{v}^{N_{v} / 2} \int_{0}^{\infty} \exp \left(-N_{v} \pi y_{v}\left(\left|\mu_{v}\right|^{2} t_{v}+\left|\nu_{v}^{\prime}\right|^{2} / t_{v}\right) t_{v}^{N_{v} S} \frac{d t_{v}}{t_{v}}\right. \\
& =y_{v}^{N_{v} / 2}\left|\frac{\nu_{v}^{\prime}}{\mu_{v}}\right|^{N_{v} s} K_{N_{v} s}\left(N_{v} \pi y_{v}\left|\mu_{v} \nu_{v}^{\prime}\right|\right) .
\end{aligned}
$$


Note that the product

$$
\prod_{v} B_{v}\left(\mu, \nu^{\prime}, y, s\right)=\mathbf{N} y^{1 / 2}\left(\frac{\mathbf{N}\left(\nu^{\prime}\right)}{\mathbf{N}(\mu)}\right)^{s} \prod_{v} K_{N_{v}}\left(N_{v} \pi y_{v}\left|\mu_{v} \nu_{v}^{\prime}\right|\right)
$$

depends only on the equivalence class $\left[\mu, \nu^{\prime}\right]$, because the absolute value of the norm of a unit is equal to 1 .

We let $R(\mathfrak{a})$ be a set of representatives of elements $\neq 0$ in $\mathfrak{a}$ for the equivalence

$$
\mu_{1} \sim \mu \text { if and only if } \mu_{1}=\epsilon \mu \text { for some unit } \epsilon \in \mathfrak{o} \text {. }
$$

The sum over $(\mu)$ in the definition of $\zeta(s, \mathfrak{a})$ could also be taken for $\mu \in R(\mathfrak{a})$.

We also define the fudge factor at infinity

$$
\begin{aligned}
G_{F, \infty}(s) & =\left(\pi^{-s / 2} \Gamma(s / 2)\right)^{r_{1}}\left((2 \pi)^{-s} \Gamma(s)\right)^{r_{2}} \\
G_{F, \infty}(2 s) & =\prod_{v}\left(N_{v} \pi\right)^{-N_{v} s} \Gamma\left(N_{v} s\right) .
\end{aligned}
$$

The full fudge factor is

$$
G_{F}(s)=\mathbf{D}(\mathfrak{o})^{s / 2} G_{F, \infty}(s)
$$

with the discriminant appearing at the finite places. Thus

$$
G_{F}(2 s)=\mathbf{D}^{s}\left(\pi^{-s} \Gamma(s)\right)^{r_{1}}\left((2 \pi)^{-2 s} \Gamma(2 s)\right)^{r_{2}} .
$$

This factor will be the relevant one for the Eisenstein series.

We recall the functional equation for $\zeta(s, \mathfrak{a})$. Let $\mathfrak{a}^{\prime}$ be the complementary fractional ideal, that is $\mathfrak{a}^{\prime}=\mathfrak{d}^{-1} \mathfrak{a}^{-1}$, where $\mathfrak{d}$ is the different. Then

$$
G_{F}(s) \zeta(s, \mathfrak{a})=G_{F}(1-s) \zeta\left(1-s, \mathfrak{a}^{\prime}\right)
$$

For the next theorem, we define the set of equivalence classes:

$$
\begin{gathered}
{[\mathrm{Equ}]\left(\mathfrak{a}, \mathfrak{a}^{\prime}\right)=\text { equivalence classes of pairs }\left[\mu, \nu^{\prime}\right] \text { with }} \\
\\
\mu \in \mathfrak{a}, \nu^{\prime} \in \mathfrak{a}^{\prime}, \text { and } \mu \nu^{\prime} \neq 0 .
\end{gathered}
$$

We let $S$ be the trace of $F / \mathbf{Q}$ as usual. 
TheOREM 3.1. The Eisenstein series have an expression:

$$
\begin{aligned}
& E(z, s, \mathfrak{a})=\mathbf{N} y(z)^{s} \zeta(2 s, \mathfrak{a})+\mathbf{N} y(z)^{1-s} \\
& \quad+\mathbf{D}^{-1 / 2} G_{F, \infty}(2 s)^{-1} G_{F, \infty}(2 s-1) \zeta(2 s-1, \mathfrak{a}) \\
& \quad+\mathbf{D}^{-1 / 2} G_{F, \infty}(2 s)^{-1} \mathbf{N a}^{2 s-1} \sum_{\left[\mu, \nu^{\prime}\right]} e^{2 \pi i S\left(\mu \nu^{\prime} x\right)} \prod_{v} B_{v}\left(\mu, \nu^{\prime}, y, s-\frac{1}{2}\right),
\end{aligned}
$$

where the sum is taken for $\left[\mu, \nu^{\prime}\right] \in[\mathrm{Equ}]\left(\mathfrak{a}, \mathfrak{a}^{\prime}\right)$. The first two terms have the meromorphic continuation coming from the Dedekind zeta function, and the sum over $\left[\mu, \nu^{\prime}\right]$ is entire in $s$.

Proof. We decompose the sum in (1) as follows:

$$
\sum_{\{\mu, \nu\}}=\sum_{\substack{\nu \in R(\mathfrak{a}) \\ \mu=0}}+\sum_{\mu \in R(\mathfrak{a})} \sum_{\nu \in \mathfrak{a}} .
$$

Then for $\operatorname{Re}(s)>1$ we get:

$$
\begin{aligned}
& E(z, s, \mathfrak{a})=\sum_{\nu \in R(\mathfrak{a})} \mathbf{N} y(0, \nu ; z)^{s} \mathbf{N a}^{2 s}+\sum_{\mu \in R(\mathfrak{a})} \sum_{\nu \in \mathfrak{a}} \mathbf{N} y(\mu, \nu ; z)^{s} \mathbf{N} \mathfrak{a}^{2 s} \\
& =\mathbf{N} y(z)^{s} \zeta(2 s, \mathfrak{a})+ \\
& \sum_{\mu \in R(\mathfrak{a})} \sum_{\nu \in \mathfrak{a}} \prod_{v}\left(N_{v} \pi\right)^{N_{v} s} \Gamma\left(N_{v} s\right)^{-1} \int_{0}^{\infty} \exp \left(-\pi N_{v} t_{v} y\left(\mu_{v}, \nu_{v} ; z_{v}\right)^{-1}\right) t_{v}^{N_{v} s} \frac{d t_{v}}{t_{v}} \mathbf{N a}^{2 s} .
\end{aligned}
$$

We now let

$$
T=\prod_{v} T_{v} \quad \text { with } \quad T_{v}=\mathbf{R}^{+}, \quad \text { and } \quad d^{*} t_{v}=\frac{d t_{v}}{t_{v}} .
$$

Thus $t=\left(\ldots, t_{v}, \ldots\right)$ is the variable in $T$. With this notation, we have

$$
\begin{aligned}
& E(z, s, \mathfrak{a})=\mathbf{N} y(z)^{s} \zeta(2 s, \mathfrak{a})+ \\
& G_{F, \infty}(2 s)^{-1} \sum_{\mu \in R(\mathfrak{a})} \int_{T} \exp \left(-\pi \operatorname{Tr}\left(t y|\mu|^{2}\right) \Theta\left(y^{-1} t, \mathfrak{a}+\mu x\right) \mathbf{N} t^{s} d^{*} t \mathbf{N a} \mathfrak{a}^{2 s},\right.
\end{aligned}
$$

where for $x=\left(\ldots, x_{v}, \ldots\right)$ we define the Hecke theta series

$$
\Theta(c, \mathfrak{a}+x)=\sum_{\alpha \in \mathfrak{a}} \exp \left(-\pi \sum_{v} N_{v} c_{v}\left|\alpha_{v}+x_{v}\right|^{2}\right) .
$$


By the Poisson summation formula-Hecke inversion (Cf. [La 70/94], Chapter XIII, §2) we get

$$
\begin{aligned}
& \Theta\left(y^{-1} t, \mathfrak{a}+\mu x\right) \\
& \quad=\mathbf{D}(\mathfrak{a})^{-1 / 2} \mathbf{N} y(z)^{1 / 2} \mathbf{N} t^{-1 / 2} \sum_{\nu^{\prime} \in \mathfrak{a}^{\prime}} e^{2 \pi i S\left(\mu \nu^{\prime} x\right)} \exp \left(-\pi \operatorname{Tr}\left(y t^{-1}\left|\nu^{\prime}\right|\right)\right) .
\end{aligned}
$$

We have $\mathbf{D}(\mathfrak{a})^{-1 / 2}=\mathbf{D}^{-1 / 2} \mathbf{N a}^{-1}$. We can write the double sum over $\mu, \nu^{\prime}$ as

$$
\sum_{\mu \in R(\mathfrak{a})} \sum_{\nu^{\prime} \in \mathfrak{a}}=\sum_{\substack{\mu \in R(\mathfrak{a}) \\ \nu^{\prime}=0}}+\sum_{\left[\mu, \nu^{\prime}\right]}
$$

The sum over $\mu \in R(\mathfrak{a})$ on the right, with $\nu^{\prime}=0$, is again a Mellin transform. The factors involving $\mathbf{N} y(z)^{1 / 2}$ and $\mathbf{N} t^{-1 / 2}$ have the effect of translating $s$ by $-1 / 2$. The sum over $\left[\mu, \nu^{\prime}\right]$ is a Bessel sum. Both these sums are the ones stated in the theorem, which is proved.

If we multiply both sides of Theorem 3.1 with $\mathbf{D}^{s} G_{F, \infty}(2 s)=G_{F}(2 s)$, we obtain a more symmetric expression as in the next theorem. In addition, it is convenient to introduce the usual abbreviations, namely we let

$$
\xi_{E}(z, s, \mathfrak{a})=G_{F}(2 s) E(z, s, \mathfrak{a}) \quad \text { and } \quad \xi_{F}(s, \mathfrak{a})=G_{F}(s) \zeta_{F}(s, \mathfrak{a}) .
$$

TheOREM 3.2. We have the expression:

$$
\begin{aligned}
& \xi_{E}(z, s, \mathfrak{a}) \\
& =\mathbf{N} y(z)^{s} \xi_{F}(2 s, \mathfrak{a})+\mathbf{N} y(z)^{1-s} \xi_{F}(2 s-1, \mathfrak{a})+ \\
& \mathbf{D}(\mathfrak{a})^{s-1 / 2} \sum_{\left[\mu, \nu^{\prime}\right]}\left(\frac{\mathbf{N}\left(\nu^{\prime}\right)}{\mathbf{N}(\mu)}\right)^{s-1 / 2} e^{2 \pi i S\left(\mu \nu^{\prime} x\right)} \prod_{v} y_{v}^{N_{v} / 2} K_{N_{v}(s-1 / 2)}\left(N_{v} \pi y_{v}\left|\mu_{v} \nu_{v}^{\prime}\right|\right) .
\end{aligned}
$$

Furthermore, we have the functional equation

$$
\xi_{E}(z, s, \mathfrak{a})=\xi_{E}\left(z, 1-s, \mathfrak{a}^{\prime}\right) .
$$

Proof. Let us replace $s$ by $1-s$ on the right side. Then by the functional equation

$$
G(w) \zeta(w, \mathfrak{a})=G(1-w) \zeta\left(1-w, \mathfrak{a}^{\prime}\right),
$$

with $w=2-2 s$, we find the term with $2 s-1$ on the right and $\mathfrak{a}$ replaced by $\mathfrak{a}^{\prime}$. A similar process starting with this term yields the first term with 
$2 s$ and also with $\mathfrak{a}$ replaced with $\mathfrak{a}^{\prime}$. Thus sending $s \mapsto 1-s$ interchanges the first two terms on the right, while replacing $\mathfrak{a}$ by $\mathfrak{a}^{\prime}$.

As to the Bessel sum, we use the functional equation of the Bessel function

$$
K_{-w}=K_{w}
$$

with $w=s-1 / 2$, changed into $-w$ by $s \mapsto 1-s$. The products $\mu \nu^{\prime}$ are unchanged when we interchange $\mathfrak{a}$ and $\mathfrak{a}^{\prime}$. The factors

$$
\mathbf{D}(\mathfrak{a})^{s-1 / 2} \text { and }\left(\mathbf{N}\left(\nu^{\prime}\right) / \mathbf{N}(\mu)\right)^{s-1 / 2}
$$

go to their inverses. Since $\mathbf{D}(\mathfrak{a})^{-1}=\mathbf{D}\left(\mathfrak{a}^{\prime}\right)$ and the pairs $\left[\mu, \nu^{\prime}\right]$ go to $\left[\nu^{\prime}, \mu\right]$, while $\mathfrak{a}^{\prime \prime}=\mathfrak{a}$, the Bessel sum also gets transformed to the corresponding Bessel sum with $\mathfrak{a}$ replaced by $\mathfrak{a}^{\prime}$. This concludes the proof.

From Theorem 3.2, we may read the residue and constant term for the Laurent expression at $s=1$ We have:

$$
\begin{aligned}
& \mathbf{N} y^{1-s}=1-(\log \mathbf{N} y)(s-1)+O\left(|s-1|^{2}\right) \\
& \xi_{F}(2 s-1, \mathfrak{a})=\frac{1}{2} \operatorname{res}_{s=1} \xi_{F}(s, \mathfrak{a}) \frac{1}{s-1}+\mathrm{CT}_{s=1} \xi_{F}(s, \mathfrak{a})+O(|s-1|),
\end{aligned}
$$

where $\mathrm{CT}_{s=1}$ denotes the constant term in a Laurent expansion about $s=1$. Therefore at $s=1$, the function $\xi_{E}(z, s, \mathfrak{a})$ has only a simple pole and the first terms of the expansion are as in the next theorem. We introduce the following functions.

The Bessel series $\operatorname{Bess}(z, \mathfrak{a})$, defined by

$$
\operatorname{Bess}(z, \mathfrak{a})=\mathbf{D}(\mathfrak{a})^{\frac{1}{2}} \sum_{\left[\mu, \nu^{\prime}\right]}\left(\frac{\mathbf{N}\left(\nu^{\prime}\right)}{\mathbf{N}(\mu)}\right)^{1 / 2} e^{2 \pi i S\left(\mu \nu^{\prime} x\right)} \prod y_{v}^{N_{v} / 2} K_{N_{v} / 2}\left(N_{v} \pi y_{v}\left|\mu_{v} \nu_{v}^{\prime}\right|\right),
$$

where the sum is taken over $\left[\mu, \nu^{\prime}\right] \in[\mathrm{Equ}]\left(\mathfrak{a}, \mathfrak{a}^{\prime}\right)$.

The Asai function $h_{F}(z, \mathfrak{a})$, defined by

$$
\underset{s=1}{\operatorname{res}} \xi_{F}(s, \mathfrak{a}) h_{F}(z, \mathfrak{a})=\xi_{F}(2, \mathfrak{a}) \mathbf{N} y+\operatorname{Bess}(z, \mathfrak{a}) .
$$

TheOREm 3.3. At $s=1$, the expansion of the Eisenstein series is given by:

$$
\xi_{E}(z, s, \mathfrak{a})=\underset{s=1}{\operatorname{res}} \xi_{F}(s, \mathfrak{a})\left[\frac{1 / 2}{s-1}-\frac{1}{2} \log \mathbf{N} y+h_{F}(z, \mathfrak{a})\right]+O(|s-1|) .
$$

Note. Our normalization of the Asai function is $1 / 2$ that of Asai's normalization in [As 70]. 


\section{$\S 4$. Modularity of $h_{F}(z, \mathfrak{a})$}

Before dealing with $h_{F}(z, \mathfrak{a})$, we mention some properties of the Eisenstein series which will now become relevant. First an invariance property. We let:

$$
\begin{aligned}
\mathbf{G}_{v} & =\mathrm{SL}_{2}(\mathbf{R}) \text { if } v \text { is real, and } \mathrm{SL}_{2}(\mathbf{C}) \text { if } v \text { is complex; } \\
\mathbf{G}_{\mathbf{F}, \infty} & =\prod_{v \in S_{\infty}} \mathbf{G}_{v}
\end{aligned}
$$

We let $\mathfrak{o}=\mathfrak{o}_{F}$ be the ring of algebraic integers of $F$. Then $\mathrm{SL}_{2}(\mathfrak{o})$ gets imbedded into $\mathbf{G}_{v}$ for each $v$ by our fixed imbedding of $\mathfrak{o}$ into $\mathbf{R}$ or $\mathbf{C}$ corresponding to $v$, so we have an imbedding

$$
\mathrm{SL}_{2}(\mathfrak{o}) \hookrightarrow \mathbf{G}_{F, \infty}
$$

on the diagonal. An element $\sigma \in \mathrm{SL}_{2}(\mathfrak{o})$ will be identified with its image, so we can write $\sigma$ as a vector

$$
\sigma=\left(\ldots, \sigma_{v}, \ldots,\right)
$$

where $\sigma_{v}$ is the image of $\sigma$ in $\mathbf{G}_{v}$. It is immediate that $\operatorname{SL}_{2}(\mathfrak{o})$ is a discrete subgroup of $G_{F, \infty}$, which we also denote by $\Gamma$.

The group $\mathbf{G}_{F}$ operates on $\mathbf{h}_{F}$ via diagonal action and then operates as a discrete subgroup on $\mathbf{h}_{F}$.

Let $\mathfrak{a}$ be a fractional ideal $\neq(0)$. Let

$$
\begin{aligned}
& \mathbf{S}(F, \mathfrak{a})=\text { the set of all matrices } \\
& \sigma=\left(\begin{array}{cc}
\xi & \eta \\
\mu & \nu
\end{array}\right) \in \mathrm{SL}_{2}(F) \quad \text { such that }(\mu, \nu)=\mathfrak{a}, \\
& \mathbf{S}_{\infty}(F, \mathfrak{o})=\text { subgroup of } \mathrm{SL}_{2}(F) \text { consisting of matrices of the form } \\
& \left(\begin{array}{cc}
\varepsilon^{-1} & \lambda \\
0 & \varepsilon
\end{array}\right) \quad \text { with } \lambda \in F \text { and a unit } \varepsilon \text {. }
\end{aligned}
$$

Then we have a bijection

$$
\mathbf{S}_{\infty}(F, \mathfrak{o}) \backslash \mathbf{S}(F, \mathfrak{a}) \longrightarrow \operatorname{Equ}^{*}(\mathfrak{a})
$$

which to each matrix in $\mathbf{S}(F, \mathfrak{a})$ as above associates the pair $(\mu, \nu)$.

Note that $\mathrm{SL}_{2}(\mathfrak{o})$ operates on the right of $\mathrm{Equ}^{*}(\mathfrak{a})$, in a manner corresponding to matrix multiplication on the right of $\mathbf{S}(F, \mathfrak{a})$. 
With the above notation, we may then express the primitive Eisenstein series as a sum

$$
E^{*}(z, s, \mathfrak{a})=\sum_{\sigma \in \mathbf{S}_{\infty} \backslash \mathbf{S}(\mathfrak{a})} \mathbf{N} y(\sigma\langle z\rangle)^{s} \mathbf{N a}^{2 s},
$$

where we abbreviated $\mathbf{S}_{\infty}(F, \mathfrak{o})=\mathbf{S}_{\infty}$ and $\mathbf{S}(F, \mathfrak{a})=\mathbf{S}(\mathfrak{a})$.

Although strictly speaking, we shall not need the following remarks leading to Proposition 4.1, and they are essentially well-known, we include them here for the convenience of the reader. They have to do with the operation of $\mathrm{SL}_{2}(\mathfrak{o})$ on the projective line $\mathbf{P}^{1}(F)$, and have to do with the compactification of $\mathrm{SL}_{2}(\mathfrak{o}) \backslash \mathbf{h}_{F}$ by the cusps, which correspond to the ideal classes, but we do not go into these considerations here.

The group $\mathrm{SL}_{2}(F)$ operates on the projective line $\mathbf{P}^{1}(F)$ as usual. If $z \in F$ and

$$
M=\left(\begin{array}{cc}
\xi & \eta \\
\mu & \nu
\end{array}\right) \in \operatorname{SL}_{2}(F),
$$

then the operation is given by $z \mapsto(\xi x+\eta) /(\mu z+\nu)$, with the possible value $\infty$. We may also represent this operation on vectors

$$
\left(\begin{array}{l}
\alpha_{1} \\
\alpha_{2}
\end{array}\right) \longmapsto M\left(\begin{array}{l}
\alpha_{1} \\
\alpha_{2}
\end{array}\right)
$$

where $\left(\begin{array}{c}\alpha_{1} \\ \alpha_{2}\end{array}\right)$ represents the element $\alpha=\alpha_{1} / \alpha_{2} \in F \cup\{\infty\}$.

To each $\alpha \in F, \alpha \neq 0$ we associate an ideal class $c_{\alpha}$ as follows. We write $\alpha=\alpha_{1} / \alpha_{2}$ with $\alpha_{1}, \alpha_{2} \in \mathfrak{o}, \alpha_{1} \alpha_{2} \neq 0$, and we let $\mathfrak{a}=\left(\alpha_{1}, \alpha_{2}\right)$ be the ideal generated by $\alpha_{1}$ and $\alpha_{2}$. If we write $\alpha=\beta_{1} / \beta_{2}$ with $\beta_{1}, \beta_{2} \in \mathfrak{o}$, then there exists $\lambda \in F^{*}$ such that

$$
\mathfrak{b}=\lambda \mathfrak{a}, \quad \text { where } \quad \mathfrak{b}=\left(\beta_{1}, \beta_{2}\right),
$$

namely $\lambda=\beta_{2} \alpha_{2}^{-1}$, so the ideal class $c_{\alpha}$ of $\mathfrak{a}$ is well defined.

Proposition 4.1. The association $\alpha \mapsto c_{\alpha}$ induces a bijection between orbits of $\mathrm{SL}_{2}(\mathfrak{o})$ in $F^{*}$ and the set of ideal classes of $F$.

The proof is routine and well-known. Cf. for instance [Si 61/80], Chapter III, §1, Proposition 20, and [Ge 80], Chapter I, Proposition 1.1. But the matter is older, cf. Siegel's comment p. 207: "It was Blumenthal who first 
gave a method of constructing a fundamental domain for $\Gamma$ in $\mathfrak{H}_{n}$, but his proof contained an error since he obtained a fundamental domain with just one cups and not $h$ cusps. This was set right by Maass."

Proposition 4.2. The Eisenstein series are invariant under the action of $\mathrm{SL}_{2}(\mathfrak{o})$, that is for $\sigma \in \mathrm{SL}_{2}(\mathfrak{o})$ and any non-zero fractional ideal $\mathfrak{a}$, we have

$$
E^{*}(\sigma\langle z\rangle, s, \mathfrak{a})=E^{*}(z, s, \mathfrak{a}),
$$

and similarly when $E^{*}$ is replaced by $E$.

Proof. It is clear that the result for $E^{*}$ implies the result for $E$. As for $E^{*}$, the result comes from the fact that multiplication by $\sigma$ on the right permutes the elements of $\mathbf{S}_{\infty} \backslash \mathbf{S}(\mathfrak{a})$.

$$
\begin{gathered}
\text { Theorem 4.3. For } \sigma \in \mathrm{SL}_{2}(\mathfrak{o}), \sigma=\left(\begin{array}{cc}
* & * \\
\gamma & \delta
\end{array}\right) \text {, let } \\
J_{v}(\sigma, z)=\left|\gamma_{v} x_{v}+\delta_{v}\right|^{2}+\left|\gamma_{v}\right|^{2} y_{v}^{2},
\end{gathered}
$$

and

$$
\mathbf{N} J(\sigma, z)=\prod_{v} J_{v}(\sigma, z)^{N_{v}} .
$$

Then $h_{F}(z, \mathfrak{a})$ satisfies the modular relation

$$
h_{F}(\sigma\langle z\rangle, \mathfrak{a})+\frac{1}{2} \log \mathbf{N} J(\sigma, z)=h_{F}(z, \mathfrak{a}) .
$$

Proof. Let $a_{-1}$ and $a_{0}(z)$ denote the residue and constant term respectively for the Eisenstein series $E(z, s, \mathfrak{a})$, so

$$
E(z, s, \mathfrak{a})=\frac{a_{-1}}{s-1}+a_{0}(z)+O(|s-1|) .
$$

From Proposition 4.2, we conclude that for $\sigma \in \mathrm{SL}_{2}(\mathfrak{o})$,

$$
a_{0}(\sigma\langle z\rangle)=a_{0}(z) .
$$

Up to a constant factor $C$, by Theorem 3.3 , we know that

$$
C a_{0}(z)=-\frac{1}{2} \log \mathbf{N} y+h_{F}(z, \mathfrak{a}) .
$$

Applying (2) proves the theorem. 
Finally, we want to apply the transformation theory of $\S 1, \S 2$ to the present case, as does Asai, so we restrict $h_{F}(z, \mathfrak{a})$ to $z=y$ so then $x=0$, which we write as $h_{F}(y, \mathfrak{a})$. We let

$$
\begin{aligned}
c_{-1}(\mathfrak{a}) & =\operatorname{res}_{s=1} \xi_{F}(s, \mathfrak{a}) \\
h_{0}(y, \mathfrak{a}) & =c_{-1}(\mathfrak{a})^{-1} \mathbf{D}(\mathfrak{a})^{1 / 2} \sum_{\left[\mu, \nu^{\prime}\right]}\left(\frac{\mathbf{N}\left(\nu^{\prime}\right)}{\mathbf{N}(\mu)}\right)^{1 / 2} \mathbf{N} y^{1 / 2} \prod K_{N_{v} / 2}\left(N_{v} \pi y_{v}\left|\mu_{v} \nu_{v}^{\prime}\right|\right), \\
& =c_{-1}(\mathfrak{a})^{-1} \operatorname{Bess}(y, \mathfrak{a}),
\end{aligned}
$$

where the sum is taken over $\left[\mu, \nu^{\prime}\right] \in[\mathrm{Equ}]\left(\mathfrak{a}, \mathfrak{a}^{\prime}\right)$. Let

$$
c_{0}=c_{0}(\mathfrak{a})=\xi_{F}(2, \mathfrak{a}) / c_{-1}(\mathfrak{a}) .
$$

THEOREM 4.4. The function $h_{0}(y, \mathfrak{a})$ has exponential decay whenever $\mathbf{N} y \rightarrow \infty$, and we have

$$
h_{F}(y, \mathfrak{a})=h_{0}(y, \mathfrak{a})+c_{0} \mathbf{N} y .
$$

Furthermore, we have the transformation rule

$$
h_{F}\left(y^{-1}, \mathfrak{a}\right)+\log \mathbf{N} y=h_{F}(y, \mathfrak{a}) .
$$

Proof. This comes from the definitions and Theorem 3.3, as well as applying Theorem 4.3 with

$$
\sigma=\left(\begin{array}{cc}
0 & -1 \\
1 & 0
\end{array}\right)
$$

\section{$\S 5$. Harmonicity of $h_{F}(z, \mathfrak{a})$}

So far we have dealt only with the algebraic properties of $h_{F}$. Now we deal with differential geometric properties and we consider the behavior of $E(z, s, \mathfrak{a})$ vis a vis the Laplace operators corresponding to each factor.

The metric form on $\mathbf{h}_{3}$ is represented by

$$
\frac{1}{y^{2}}\left(d x_{1}^{2}+d x_{2}^{2}+d y^{2}\right)
$$

and the corresponding volume form by $d x_{1} d x_{2} d y / y^{2}$. The formulas for $\mathbf{h}_{2}$ are even better known. The positive Laplace operator on $\mathbf{h}_{2}$ is given by

$$
\boldsymbol{\Delta}_{2}=-y^{2}\left(\frac{\partial^{2}}{\partial x^{2}}+\frac{\partial^{2}}{\partial y^{2}}\right)
$$


The positive Laplacian $\boldsymbol{\Delta}_{3}$ on $\mathbf{h}_{3}$ is given by

$$
\begin{aligned}
\boldsymbol{\Delta}_{3} & =-y^{2}\left(4 \frac{\partial^{2}}{\partial x \partial \bar{x}}+\frac{\partial^{2}}{\partial y^{2}}\right)+y \frac{\partial}{\partial y} \\
& =-y^{2}\left(\frac{\partial^{2}}{\partial x_{1}^{2}}+\frac{\partial^{2}}{\partial x_{2}^{2}}+\frac{\partial^{2}}{\partial y^{2}}\right)+y \frac{\partial}{\partial y}
\end{aligned}
$$

It is the unique (up to constant factor) $\mathrm{SL}_{2}(\mathbf{C})$-invariant differential operator on $\mathbf{h}_{3}$. For each absolute value $v$, we let $\boldsymbol{\Delta}_{v}$ be the corresponding Laplacian.

We can exhibit eigenfunctions of the Laplace operator as follows. Let $\alpha \neq 0$ be a complex number and let

$$
e_{s}(\alpha, z)=y K_{2 s-1}(|\alpha| y) e^{2 i \operatorname{Re}(\alpha x)}
$$

Note that if we denote by $e_{s}^{B}(\alpha, z)$ the corresponding function in Asai, then his normalization is related to ours by $2 e_{s}^{B}(\alpha, z)=e_{s}(\alpha, z)$.

Proposition 5.1. We have the eigenfunctions

$$
\boldsymbol{\Delta}_{v} y_{y}^{N_{v} s}=N_{v}^{2} s(1-s) y_{v}^{N_{v} s} \quad \text { and } \quad \boldsymbol{\Delta}_{v} e_{s}(\alpha, z)=N_{v} s(1-s) e_{s}(\alpha, z) .
$$

Proof. The first formula follows from a direct computation. The second one is a consequence of the differential equation satisfied by the $K$-Bessel function $K_{s}(y)$, which is standard, and is derived directly from our definition of the $K$-Bessel function in [JoL 96], Lemma 3.1.

From (1) in $\S 4$, applying the above eigenfunction relation to each term in the sum, and to all fractional ideals $\mathfrak{a b}$ with $\mathfrak{b}$ integral $\neq 0$, we find:

Proposition 5.2. For all $v$ and all Laplacians $\boldsymbol{\Delta}_{v}$ we have

$$
\boldsymbol{\Delta}_{v} E(z, s, \mathfrak{a})=N_{v}^{2} s(1-s) E(z, s, \mathfrak{a}),
$$

and similarly for $E^{*}$ instead of $E$.

We then obtain the corresponding result for the function $h_{F}(z, \mathfrak{a})$.

Proposition 5.3. This function is harmonic for each $\boldsymbol{\Delta}_{v}$, that is

$$
\boldsymbol{\Delta}_{v} h_{F}(z, \mathfrak{a})=0
$$


Proof. Let $a_{-1}$ and $a_{0}(z)$ be as in the proof of Theorem 4.3. From Proposition 5.2 we find

$$
\boldsymbol{\Delta}_{v} E(z, s, \mathfrak{a})=-N_{v}^{2} a_{-1}+O(|s-1|),
$$

since the right side of the first formula in Proposition 5.1 contains a factor $-(s-1)$. But we also have

$$
\boldsymbol{\Delta}_{v} E(z, s, \mathfrak{a})=\boldsymbol{\Delta}_{v} a_{0}(z)+O(|s-1|)
$$

Therefore

$$
\boldsymbol{\Delta}_{v} a_{0}(z)=-N_{v}^{2} a_{-1} .
$$

Furthermore, by direct computation,

$$
\boldsymbol{\Delta}_{v} \log \mathbf{N} y(z)=N_{v}^{2} .
$$

Hence

$$
\boldsymbol{\Delta}_{v}\left(a_{0}(z)+a_{-1} \log \mathbf{N} y(z)\right)=0 .
$$

But from Theorem 3.3, one sees that

$$
a_{0}(z)+a_{-1} \log \mathbf{N} y(z)
$$

up to a constant factor is equal to

$$
-\frac{1}{2} \log \mathbf{N} y+h_{F}(z, \mathfrak{a})+\frac{1}{2} \log \mathbf{N} y .
$$

This proves that $\boldsymbol{\Delta}_{v} h_{F}(z, \mathfrak{a})=0$, and concludes the proof of the proposition.

\section{$\S 6$. Regularized products}

We show here that the Eisenstein series give examples of regularized products, as defined in [JoL 93a] and [JoL 93b]. See also the definition of regularized product type in [JoL 94], Chapter I, §6. To save space, we do not reproduce the relevant definitions here.

TheOREm 6.1. For each $z$ and $\mathfrak{a}$, the Eisenstein functions $E^{*}(z, s, \mathfrak{a})$, $E(z, s, \mathfrak{a})$ are of regularized product type. So are $E_{F}^{*}(z, s)$ and $E(z, s)$. Furthermore, $E(z, s, \mathfrak{a})$ is of order 1 and has polynomial growth in vertical strips. 
Proof. We first prove the property of regularized product type for $E(z, s, \mathfrak{a})$. This property is actually a corollary of our general Cramér theorem from [JoL 93b]. It suffices to verify the conditions of this theorem. By combining equation (1) in $\S 4$ and (6) in $\S 3$, we see that $E(z, s, \mathfrak{a})$ has a Dirichlet series representation in a right half plane (see also (1) in $\S 3$ ). By Theorem 3.2, the function

$$
\xi_{E}(z, s, \mathfrak{a})=G_{F}(2 s) E(z, s, \mathfrak{a})
$$

satisfies a functional equation, actually is invariant under $s \mapsto 1-s$. Since $G_{F}$ is of regularized product type, it remains to show that $E(z, s, \mathfrak{a})$ is of finite order, actually order 1 , and has polynomial growth in vertical strips. This is done by classical routine arguments as follows.

Since $E(z, s, \mathfrak{a})$ has a Dirichlet series representation in a right half plane, it is bounded in a slightly smaller right half plane. By the functional equation, we have

$$
E(z, 1-s, \mathfrak{a})=G_{F}(2-2 s)^{-1} G(2 s) E(z, s, \mathfrak{a}) .
$$

Since $G_{F}(2 s) / G_{F}(2-2 s)$ has a polynomial growth in vertical strips and is of order 1 , the function $E(z, s, \mathfrak{a})$ has polynomial growth in vertical strips in some left half plane and is of order 1 in this left half plane, i.e. $O\left(e^{|s|^{1+\varepsilon}}\right)$ for $|s| \rightarrow \infty, s$ in that left half plane. Observe that the proof of Theorem 3.1, or Theorem 3.2, expresses $E$ as a sum of terms involving the Dedekind zeta function and gamma function, and a Bessel series times $G_{F, \infty}(2 s)^{-1}$. For $\sigma=\operatorname{Re}(s)$ in a finite interval (so $s$ itself is in a vertical strip), the Bessel function satisfies an exponential estimate

$$
\left|K_{s}(c)\right| \leq C e^{-2 c},
$$

uniformly for $\sigma$ in the interval. The number of elements in a fractional ideal with absolute values bounded by $B$ (with $B \rightarrow \infty$ ) is polynomial in $B$, and the absolute values of elements in such a $\mathbf{Z}$-lattice are bounded from below. The elements can be split up into annuli according to the maximum absolute value, and one can estimate the Bessel series by an integral

$$
\int_{\delta}^{\infty} \int_{\delta}^{\infty} x^{ \pm M} y^{ \pm M^{\prime}} e^{-c x y} d x d y, \quad \text { with some } c, M, M^{\prime}>0
$$


and with some fixed $\delta>0$. In the application, $M, M^{\prime}$ are bounded when $\sigma$ lies in a finite interval. Hence, the Bessel series is bounded in the strip.

The factor $G_{F}(2 s)^{-1}$ has order 1 in vertical strips. Theorem 3.2 shows that the function $\xi_{E}$ (as a function of $s$ ) is entire, except for trivial poles of $\xi_{F}$. Hence, the Eisenstein function has only a finite number of poles in a given vertical strip. Thus, we have proved that the Eisenstein function is of order 1. Finally, the functional equation

$$
E(1-s)=\Phi(s) E(s)
$$

with a fudge factor $\Phi(s)$ which is a quotient of gamma factors shows that $E$ has polynomial growth on vertical lines far to the right and to the left. Since it has order 1, we can apply the Phragmen-Lindelöf theorem to see that it has polynomial growth in vertical strips. This concludes the proof.

Note that the fudge factors in the functional equation of the Eisenstein functions involve both gamma factors and zeta factors with the Riemann zeta function. Thus we are already on the third rung of the ladder of functions which are regularized products.

Note also that the functional equations are multiplicative and we are not dealing with the logarithmic derivatives, which are of regularized harmonic series type.

The Eisenstein functions satisfy not only the ladder theorem, but they satisfy conditions which allow us to get an asymptotic expansion for the associated theta series. More precisely, combining (1) from $\S 4$ and the Dirichlet series representation of $\zeta_{F}(s, \mathfrak{a})$ we obtain a Dirichlet series representation for $E(z, s, \mathfrak{a})$, which we write in the form

$$
\begin{aligned}
E(z, s, \mathfrak{a}) & =\zeta_{F}(s, \mathfrak{a}) E^{*}(z, s, \mathfrak{a}) \\
& =\mathbf{N a}^{s} \sum_{(\mu)} \mathbf{N}(\mu)^{-s} \sum_{\mathbf{S}_{\infty} \backslash \mathbf{S}(\mathfrak{a})} \mathbf{N} y(\sigma\langle z\rangle)^{s} \\
& =\sum_{k} a_{k} \lambda_{k}^{-s} .
\end{aligned}
$$

Thus we have the associated theta series defined by

$$
\Theta(z, t, \mathfrak{a})=\Theta(t)=\sum a_{k} e^{-\lambda_{k} t} .
$$

In [JoL 93a], [JoL 93b] and [JoL 94], we have systematically used the three axioms AS 1, AS 2, AS 3, of which the main axiom is AS 2, asserting 
the existence of an asymptotic expansion for $\Theta(t)$ at the origin, in terms of generalized polynomials. In [JoL 93a], we gave a criterion to get such an asymptotic expansion. We can now use this criterion to prove:

THEOREM 6.2. The theta series $\Theta(z, t, \mathfrak{a})$ satisfies the asymptotic conditions AS 1, AS 2 and AS 3.

Proof. The result comes from a direct application of [JoL 93a], $§ 7$. By the polynomial growth asserted in Theorem 6.1, the Eisenstein series $E(z, s, \mathfrak{a})$ is in the domain of the inverse Mellin transform. Hence, Theorem 7.4 and Theorem 7.5 of [JoL 93a] apply to conclude the proof of Theorem 6.2.

The above two results show how easily our axioms can be applied in certain concrete specific situations.

One can further apply the results of [JoL 94] to obtain an explicit formula and theta inversion formula associated to the Dirichlet series expansion for $E(z, s, \mathfrak{a})$. Thus one can do for the Eisenstein series themselves what we pointed out for the scattering determinant in another special case, see [JoL 96], $§ 7$.

\section{§. Heat kernel, spectral theoretic applications and associated zeta functions}

We describe how one can introduce the heat kernel to produce theta relations via a spectral decomposition and then we can apply the Gauss transform to get zeta functions, as we pointed out already in [JoL 94] and [JoL 96].

Let $\mathbf{L}_{F}$ be the heat operator on $\mathbf{h}_{F}$, acting on functions $u(t, x)$ of a real variable $t$ and variable $x \in \mathbf{h}_{F}$. Specifically, $\mathbf{L}_{F}$ acts via an operator $\mathbf{L}_{v}$ at each place $v$, and

$$
\mathbf{L}_{v}=\boldsymbol{\Delta}_{v}+N_{v}^{2} \frac{\partial}{\partial t}
$$

so

$$
\begin{aligned}
& \mathbf{L}_{v}=\boldsymbol{\Delta}_{v}+\frac{\partial}{\partial t} \quad \text { if } v \text { is real } \\
& \mathbf{L}_{v}=\boldsymbol{\Delta}_{v}+4 \frac{\partial}{\partial t} \quad \text { if } v \text { is complex. }
\end{aligned}
$$


Let $\mathbf{K}_{\mathbf{h}_{F}}$ be the heat kernel on $\mathbf{h}_{F}$, associated to the Laplacian which acts on $C_{c}^{\infty}\left(\mathbf{h}_{F}\right)$ (smooth compactly supported functions). It is immediate that

$$
\mathbf{K}_{\mathbf{h}_{F}}=\prod_{v \in S_{\infty}} \mathbf{K}_{\mathbf{h}_{v}},
$$

where $\mathbf{K}_{\mathbf{h}_{v}}$ is the heat kernel on the hyperbolic half plane $\mathbf{h}_{2}$ if $v$ is real and the heat kernel on hyperbolic $\mathbf{h}_{3}$ if $v$ is complex. We recall that

$$
e^{t / 4} \mathbf{K}_{\mathbf{h}_{2}}(t, \rho)=\frac{1}{4 \pi t} e^{-\rho^{2} / 4 t} g(t, \rho)
$$

where

$$
g(t, \rho)=t^{-1 / 2} \int_{\rho}^{\infty} \frac{u e^{\left(\rho^{2}-u^{2}\right) / 4 t}}{(\cosh u-\cosh \rho)^{1 / 2}} \frac{d u}{\sqrt{2 \pi}}
$$

and

$$
e^{t / 4} \mathbf{K}_{\mathbf{h}_{3}}(t, \rho)=\frac{1}{(\pi t)^{3 / 2}} e^{-\rho^{2} / t} \frac{\rho}{\sinh \rho} .
$$

Warning. The factor $N_{v}$ in the definition of the norm on $\mathbf{h}_{F}$ was natural (and follows Asai), since, for example, all Eisenstein series then have a functional equation which amounts to invariance under the map $s \mapsto 1-s$. Other normalizations occur in the literature. For instance, if we let $E_{\mathrm{EGM}}$ denote the Eisenstein series as defined in [EGM 85], then

$$
E_{\mathrm{EGM}}(z, s, \mathfrak{a})=E(z, s / 2, \mathfrak{a}) .
$$

Our normalization entails a scaling of the heat kernel when $v$ is complex. Note that the usual $\rho^{2} / 4 t$ (present for $\mathbf{h}_{2}$ ) becomes $\rho^{2} / t$ for $\mathbf{h}_{3}$ (the complex case) and $(4 \pi t)^{3 / 2}$ becomes $(\pi t)^{3 / 2}$. If one denotes by $\mathbf{K}_{\mathbf{R}}$ the real heat kernel, found elsewhere in the literature (e.g. [JoL 94], [JoL 96]), then

$$
\mathbf{K}_{\mathbf{C}, \mathbf{h}_{3}}(t, \rho)=\mathbf{K}_{\mathbf{h}_{3}}(t, \rho)=\mathbf{K}_{\mathbf{R}, \mathbf{h}_{3}}(\rho, t / 4) .
$$

More appropriately, we may then use the notation $\mathbf{K}_{v}$ for the heat kernel at the place $v$, where $\mathbf{K}_{v}$ is $\mathbf{K}_{\mathbf{h}_{2}}$ or $\mathbf{K}_{\mathbf{C}, \mathbf{h}_{3}}$ according as $v$ is real or complex.

Given a Hecke character $\chi=[\beta]$ (notation of $\S 1$, see the $\mathbf{O R}$ conditions), we define the corresponding primitive Hecke-Eisenstein series as in $\S 4,(1)$ by

$$
E^{*}(z, s, \mathfrak{K}, \chi)=\sum_{\sigma \in \mathbf{S}_{\infty} \backslash \mathbf{S}(\mathfrak{a})} \chi(y(\sigma\langle z\rangle)) \mathbf{N} y(\sigma\langle z\rangle)^{s} \mathbf{N a}^{2 s}
$$


where, as in $\S 2, \chi(y)=\prod y_{v}^{N_{v} \beta_{v}}=\mathbf{N}\left(y^{\beta}\right)$. By relations OR 1 and OR $\mathbf{2}$ of $\S 1$, the series (1) is, at least formally, invariant under the action of $\mathrm{SL}_{2}(\mathfrak{o})$. The formalism of $\S 1$ applies directly and similar estimates as before extend the results of $\S 3$ through $\S 6$ to the Eisenstein series with Hecke characters to yield meromorphic continuations, functional equations and Fourier expansions. These were actually carried out in the Hilbert modular case (when $F$ is totally real) in [Ef 87] and [Zo 82].

Let

$$
X_{F}=\mathrm{SL}_{2}(\mathfrak{o}) \backslash \mathbf{h}_{F} .
$$

In the Hilbert modular case, [Ef 87] and [Zo 82] also deal with the eigenfunction expansion in $L^{2}\left(X_{F}\right)$. See especially [Ef 87] p. 41, Definition 1.7, p. 83, Theorem 9.8 for one cusp and p. 100 in the general case. Similar results are valid in the general case with arbitrary number fields. The proofs are similar and we merely summarize the situation.

The space $L^{2}\left(X_{F}\right)$ has an orthogonal decomposition consisting of a discrete part and a continuous part, as follows. The discrete part is the direct sum orthogonal decomposition of the subspace of $L^{2}\left(X_{F}\right)$ generated by the eigenfunctions. The continuous part is determined by the family

$$
E^{*}\left(z, \frac{1}{2}+i r, \mathfrak{K}, \chi\right)
$$

parametrized by the ideal classes $\mathfrak{K}$ all $r \in \mathbf{R}$ and all Hecke characters $\chi$. As recalled in $\S 1$, the group of Hecke characters is isomorphic to $\mathbf{Z}^{r_{1}+r_{2}-1}$, and thus it constitutes a discrete family, but the variable $r$ parametrizes a continuous family. Note that by using $r$ to parametrize the continuous family, we give priority to the notation of the spectral gang. (Too many $r$ 's!)

In particular, we may periodize the heat kernel $\mathbf{K}_{\mathbf{h}_{F}}$ with respect to $\mathrm{SL}_{2}(\mathfrak{o})$ to get the heat kernel $\mathbf{K}_{X_{F}}$ on $X_{F}$. The following result is obtained in the Hilbert modular case in [Ef 87], III. 4, and can be proved similarly in the general case. We let $\left\{\varphi_{k}\right\}$ be total orthonormal family of eigenfunctions in $L^{2}\left(X_{F}\right)$. We let $\left\{\lambda_{k}\right\}$ be the eigenvalues and we let

$$
\mu_{k}=\lambda_{k}-1 / 4
$$

following our standard normalization. 
Theorem 7.1. Let $\mathbf{K}_{X_{F}}(t, z, w)$ be the heat kernel on $X_{F}$. Define

$$
E_{\text {tot }}^{*}(z, w, s, \chi)=\sum_{\mathfrak{K}} E^{*}(z, s, \mathfrak{K}, \chi) \overline{E^{*}(w, s, \mathfrak{K}, \chi)} .
$$

Let $r_{\chi}=r-i \beta_{\chi}$ where $\beta_{\chi}$ is the sum of the exponents of the character associated to $\chi$. Then there is a constant $c_{F}$ such that one has the expansion:

$$
\begin{aligned}
e^{t / 4} \mathbf{K}_{X_{F}}(t, z, w)= & \sum_{\gamma \in \mathrm{SL}_{2}(\mathfrak{o}) /\{ \pm 1\}} e^{t / 4} \mathbf{K}_{\mathbf{h}_{F}}(t, \gamma \tilde{z}, \tilde{w}) \\
= & \sum_{k} \varphi_{k}(z) \overline{\varphi_{k}(w)} e^{-\mu_{k} t} \\
& +c_{F} \int_{-\infty}^{\infty} \sum_{\chi} E_{\mathrm{tot}}^{*}\left(z, w, \frac{1}{2}+i r, \chi\right) e^{-r_{\chi}^{2} t} d r .
\end{aligned}
$$

The convergence of the series (i.e., the sum over all Hecke characters $\chi)$ is addressed in [Ef 87] and [Zo 82]. It is similar to the convergence of a Fourier series. No new convergence problems arise by using the complex places of the number field.

Note that the equality of the two expansions for $\mathbf{K}_{X_{F}}$ is a theta inversion formula. For a totally imaginary number field, it fits exactly our previous formalism considered in [JoL 94] and [JoL 96]. The integral involving the Eisenstein series in Theorem 7.1 above corresponds precisely to the term $E_{\Phi}(t)$ in [JoL 94], Chapter IV, Theorem 1.2; and to the term $E_{A} R_{\text {left }}$ or $E_{-a} R_{\text {right }}$ in [JoL 96], see the "Basic Assumptions" of $\S 5$. The notation in these references was not accidental. We had in mind the Eisenstein series as an eventual manifestation of such terms, which do not occur for compact quotients.

For arbitrary $F$, because of the real places, the theta inversion formula is generalized because of the integral expression for the heat kernel on the hyperbolic upper half plane. Hence our formalism has to be extended to the more general situation involving such integral representations. We have already mentioned this possibility explicitly in the above cited references. See for instance [JoL 94], Chapter V, §4, Remark 1 and [JoL 96], §8 Remark 1.

Having the theta inversion, we may proceed as in [JoL 94] and [JoL 96]. We take the Gauss transform. Let

$$
\Theta(t, z, w)=e^{t / 4} \mathbf{K}_{X_{F}}(t, z, w) .
$$


Then the Gauss transform is formally defined by

$$
\operatorname{Gauss}(\Theta)(s)=2 s \int_{0}^{\infty} e^{-s^{2} t} \Theta(t, z, w) d t .
$$

It is explained in [JoL 94] and [JoL 96] how to regularize such an integral. Then $\operatorname{Gauss}(\Theta)(s)$ is a generalized zeta-type function which admits an additive functional equation under $s \mapsto-s$ and has a generalized Bessel series representation in a right half plane. For $z \neq w$, the additive fudge term in the functional equation is expressible in terms of $E_{\mathrm{spec}}^{*}(z, w, s)$, which is the Gauss transform of

$$
c_{F} \int_{-\infty}^{\infty} \sum_{\chi} E_{\mathrm{tot}}^{*}\left(z, w, \frac{1}{2}+i r, \chi\right) e^{-r^{2} t} d r .
$$

If the number field $F$ is totally complex, then the heat kernel is split, in the terminology of [JoL 96] and hence the extra spectral integrals for $\mathbf{h}_{2}$ do not occur. In this totally complex case, the Gauss transform is equal to a Bessel series in the sense of [JoL 96], in a right half plane and hence the results of [JoL 96] apply directly.

The theta inversion formula of Theorem 7.1 comes from the heat kernel itself, before taking the trace of the heat kernel (integrating the diagonal over the manifold). As a result, we avoid various difficulties which occur because of the presence of elliptic elements in the group $\mathrm{SL}_{2}(\mathfrak{o})$ (see remarks of Gangolli [Ga 68], pp. 153 and 190). If we take $z \neq w$, then all elements in the group side of the theta inversion formula contribute to a Bessel series, or more generally to an integral of a Bessel series expansion. If we take $z=w$, then we must consider separately the case when $z$ is or is not a fixed point of some elliptic element. If $z$ is not a fixed point, then one separates out the identity element alone. If $z$ is a fixed point, then one must consider as well all elliptic elements which fix $z$. The totality of these elements, together with the integral involving $E_{\mathrm{spec}}^{*}$, form the $E_{\Phi}$ or $E_{A} R$ terms, in the notation of [JoL 94] and [JoL 96]. Further theta inversion formulas which occur from the regularized trace of the heat kernel thus do cause additional complications caused by elliptic elements.

A detailed exposition for the above results and further applications belongs to subsequent publications. 


\section{$\S 8$. Heat Eisenstein series}

To conclude this paper, let us reformulate the spectral expansion of Theorem 7.1 in terms of a new Eisenstein series which we call the heat Eisenstein series. By doing so, we obtain a spectral expansion involving only a finite number of terms representing the continuous spectrum. In fact, the number of terms is equal to the number of points at infinity, namely the class number. We are developing this approach currently in the setting of other symmetric spaces, most notably the symmetric space associated to $\mathrm{SL}_{n}(\mathbf{R})$.

In the notation of $\S 7$, let $\chi$ be any Hecke character. The space of all Hecke characters is parameterized by $\mathbf{Z}^{r_{1}+r_{2}-1}$. In other words, there is a torus $\mathbf{T}_{\mathfrak{K}}$ isomorphic to $(\mathbf{R} / \mathbf{Z})^{r_{1}+r_{2}-1}$ such that any Hecke character is an eigenfunction of the Euclidean Laplacian on $\mathbf{T}_{\mathfrak{K}}$. Let $\mathbf{K}_{\mathbf{T}_{\mathfrak{K}}}$ denote the associated heat kernel on $\mathbf{T}_{\mathfrak{K}}$, and let $p_{\mathfrak{K}}$ denote the projection which maps points in $X_{F}$ to points onto the torus $\mathbf{T}_{\mathfrak{K}}$. The map $p_{\mathfrak{K}}$ amounts to first decomposing the space $\mathbf{h}_{F}$ in terms of its Iwasawa coordinates and then projecting any point $z \in \mathbf{h}_{F}$ onto the associated quotient of a unipotent subgroup. Consider the (formal) series

$$
\begin{aligned}
& E_{\mathfrak{K}}(t, z, w, s) \\
& \quad=\mathbf{N a}^{2 s} \overline{\mathbf{N a}^{2 s}} \sum_{\sigma, \sigma^{\prime} \in \mathbf{S}_{\infty} \backslash \mathbf{S}(\mathfrak{a})} \mathbf{K}_{\mathbf{T}_{\mathfrak{K}}}\left(t, p_{\mathfrak{K}}(z), p_{\mathfrak{K}}(w)\right) \mathbf{N} y(\sigma\langle z\rangle)^{s} \overline{\mathbf{N} y\left(\sigma^{\prime}\langle w\rangle\right)^{s}},
\end{aligned}
$$

which we take to be defined for $\operatorname{Re}(s)$ sufficiently large. By taking $t \rightarrow 0$, we obtain the formal equality

$$
\lim _{t \rightarrow 0} E_{\mathfrak{K}}(t, z, w, s)=\sum_{\chi} E^{*}(z, s, \mathfrak{K}, \chi) \overline{E^{*}(w, s, \mathfrak{K}, \chi)} .
$$

This suggests that one can use the heat Eisenstein series defined in (1) to reformulate the spectral expansion obtained in Theorem 7.1. Indeed, there is considerable work which needs to be completed in the study of the heat Eisenstein series. At this point, we assert the following property of (1). The series (1) admits a meromorphic continuation to all $s \in \mathbf{C}$ and for any smooth, bounded function $\psi$ on $X_{F}$, we have, in the notation of Theorem 7.1, the spectral expansion

$$
\psi(z)=\sum_{\mathfrak{K}}\left\langle\psi, \varphi_{k}\right\rangle \varphi_{k}(z)+c_{F} \sum_{\mathfrak{K}} \lim _{t \rightarrow 0} \int_{-\infty}^{\infty}\left\langle\psi * E_{\mathfrak{K}}\right\rangle\left(t, z, \frac{1}{2}+i r\right) d r,
$$


where

$$
\left\langle\psi * E_{\mathfrak{K}}\right\rangle(t, z, s)=\int_{X_{F}} \psi(w) E_{\mathfrak{K}}(t, z, w, s) d \mu(w)
$$

denotes the usual convolution (symmetric $L^{2}$ inner product) on $X_{F}$. Various questions which naturally arise in the study of Eisenstein series, namely results analogous to those obtained in this paper, can be asked about our heat Eisenstein series and are currently under consideration as well as the verification of $(3)$.

\section{REFERENCES}

[As 70] T. Asai, On a certain function analogous to $\log \eta(z)$, Nagoya Math. J., 40 (1970), 193-211.

[Ef 87] I. Efrat, The Selberg trace formula for $P S L_{2}(\mathbf{R})^{n}$, Memoir AMS, 359 (1987).

[EfS 85] I. Efrat and P. Sarnak, The determinant of the Eisenstein matrix and Hilbert class fields, Trans. AMS, 290 (1985), 815-824.

[EGM 85] J. Elstrodt, E. Grunewald and J. Mennicke, Eisenstein series on three dimensional hyperbolic spaces and imaginary quadratic fields, J. reine angew. Math., 360 (1985), 160-213.

[EGM 87] J. Elstrodt, E. Grunewald and J. Mennicke, Zeta functions of binary hermitian forms and special vales of Eisenstein series on three-dimensional hyperbolic space, Math. Ann., 277 (1987), 655-708.

[Ga 68] R. Gangolli, On the length spectra of some compact manifolds of negative curvature, Acta Math., 121 (1986), 151-192.

[Ge 89] G. Van der Geer, Hilbert modular surfaces, Springer Verlag, 1980.

[JoL 93a] J. Jorgenson and S. Lang, Basic analysis of regularized series and products, Springer Lecture Notes 1564, 1993.

[JoL 93b] J. Jorgenson and S. Lang, On Cramér's theorem for general Euler products with functional equations, Math. Ann., 297 (1994), 383-416.

[JoL 94] J. Jorgenson and S. Lang, Explicit formulas for regularized products and series, Springer Lecture Notes 1593, 1994.

[JoL 96] J. Jorgenson and S. Lang, Extension of analytic number theory and the theory of regularized harmonic series from Dirichlet series to Bessel series, Math. Ann., 306 (1996), 75-124.

[Ku 68] T. Kubota, Über diskontinuierlicher Gruppen Picardschen Typus und zugehörige Eisensteinsche Reihen, Nagoya Math. J., 32 (1968), 259-271.

[La 73/87] S. Lang, Elliptic Functions, Addison Wesley, 1973, (second edition Springer Verlag, 1987).

[La 70/94] S. Lang, Algebraic Number Theory, Addison Wesley, 1970, (second edition Springer Verlag, 1994).

[Sa 83] P. Sarnak, The arithmetic and geometry of some hyperbolic three manifolds, Acta. Math., 151 (1983), 253-295. 
[Si 61/80] C. L. Siegel, Advanced Analytic Number Theory, Lecture Notes Tata Institute, 1961, (reprinted in book form, 1980).

[Sz 83] J. Szmidt, The Selberg trace formula for the Picard group $S L(2, Z[i])$, Acta Arith., 42 (1983), 291-424.

[Zo 82] P. Zograf, Selberg trace formula for the Hilbert modular group of a real quadratic number field, J. Soviet Math., 19 (1982), 1637-1652.

Jay Jorgenson

Department of Mathematics

Oklahoma State University

Stillwater, OK 74078

U.S.A.

Serge Lang

Department of Mathematics

Yale University Box 20-8283

New Haven, CT 06520-8283

U.S.A. 